Review

\title{
From First Line to Sequential Treatment in the Management of Metastatic Pancreatic Cancer
}

\author{
Andrés Muñoz Martín ${ }^{\bowtie}$, Manuel Hidalgo², Rafael Alvarez³, Virginia Arrazubi4 ${ }^{4}$ Joaquina Martínez-Galán ${ }^{5}$, \\ Mercedes Salgado 6 , Teresa Macarulla7, Alfredo Carrato ${ }^{8}$ \\ 1. Dpt. Medical Oncology, Hospital Universitario Gregorio Marañón, Madrid, Spain; \\ 2. Div. Medical Oncology, Beth Israel Deaconess Medical Center, Boston, USA; \\ 3. Centro Integral Oncológico Clara Campal, Hospital Universitario HM Sanchinarro, Madrid, Spain \\ 4. Dpt. Medical Oncology, Complejo Hospitalario de Navarra, Pamplona, Spain; \\ 5. Dpt. Medical Oncology, H.U. Virgen de las Nieves, Complejo Hospitalario de Granada, Granada, Spain; \\ 6. Dpt. Medical Oncology, Complejo Hospitalario Universitario de Orense, Orense, Spain; \\ 7. Dpt. Medical Oncology, Hospital Vall d’Hebrón, Barcelona, Spain; \\ 8. Dpt. Medical Oncology, Hospital Universitario Ramón y Cajal, IRYCIS, CIBERONC, Madrid, Spain \\ $\square$ Corresponding author: Andrés J. Muñoz Martín, MD, PhD. Address: C/ Doctor Esquerdo 46, Madrid 28007, Spain. Ph: +34 914269394; Fax: +34 915 737 985; \\ Email: andresmunmar@hotmail.com \\ ( ) Ivyspring International Publisher. This is an open access article distributed under the terms of the Creative Commons Attribution (CC BY-NC) license \\ (https://creativecommons.org/licenses/by-nc/4.0/). See http://ivyspring.com/terms for full terms and conditions.
}

Received: 2017.11.07; Accepted: 2018.02.14; Published: 2018.04.30

\begin{abstract}
The current management of patients with metastatic pancreatic ductal adenocarcinoma (mPDAC) is based on systemic chemotherapy. The results of the MPACT and PRODIGE clinical trials have demonstrated that the combination of nab-paclitaxel and gemcitabine (GEM) as well as FOLFIRINOX regimen result in improvement in overall survival when compared to GEM alone. Treatment guidelines now recommend either one of these two regimens as first line treatment for fit patients with mPDAC. Because no head-to-head comparison between the two regimens exists, the selection of one versus the other is based on clinical criteria. The design and eligibility criteria of these two clinical trials are dissimilar, making the results of the MPACT trial more applicable to the general population of patients with mPDAC. In addition, the combination of nab-paclitaxel and GEM is better tolerated and easier to administer in clinical practice than FOLFIRINOX. Furthermore, when the regimens are studied in comparable patient populations the efficacy results are very similar. Nanoliposomal irinotecan plus 5FU has recently demonstrated a significant increase in efficacy rates after a GEM-based treatment. Importantly, treatment of mPDAC should now be considered as a continuum care for patients who are fit, with second and even third line treatments. Different sequential treatment algorithms are proposed based on available data. In retrospective studies, patients who were managed with GEM-based regimens followed by fluoropyrimidine-based regimens appear to have the most favorable outcome.
\end{abstract}

Key words: pancreatic cancer, metastatic disease, chemotherapy, nab-paclitaxel + gemcitabine, FOLFIRINOX, sequential treatment

\section{Introduction}

Pancreatic ductal adenocarcinoma (PDAC), one of the most lethal solid tumors, is often diagnosed at advanced, metastatic, stage. As a result, most of the patients $(80-90 \%)$ are not candidates for curative surgical resection. The most common clinical factors encountered in daily practice that should be incorporated into the treatment decision-making process to optimize patient outcomes include performance and nutritional and psychological status, patient's age, pain, and other comorbidities [1-3]. Current systemic treatment of patients with metastatic (mPDAC) is based on systemic chemotherapy. During more than twenty years, single gemcitabine (GEM) was the only approved and widely used agent $[4,5]$. However, over the last few years, two additional effective regimens have shown 
clinically meaningful and statistically significant improvement in median survival resulting in a change in first-line treatment selection [6,7]. In addition, recently, new chemotherapy regimens have significantly improved survival in second line treatment $[8,9]$. The availability of different treatment options, which obviously represents an important advance, also creates uncertainty as which regimen(s) should be recommended for specific clinical situations. To address these issues, and supported by professional societies or working groups, a series of clinical guidelines have been issued [10-13].

This article reviews the different treatment options available for mPDAC patients. A thorough revision of the literature evidence as well as experts' opinion regarding sequential treatment algorithms is also presented. The manuscript is the result of the GALLgo project, an experts' consultant initiative carried out by the ECO Foundation.

\section{Treatment recommendations based on European and American Medical Oncology Societies}

Table 1 compares the recommendations made by the European Society of Medical Oncology (ESMO), NCCN (National Comprehensive Cancer Network), ASCO (American Society for Clinical Oncology) and SEOM (Sociedad Española de Oncología Médica) Guidelines, based on performance status (Eastern Cooperative Group Performance
Status, ECOG) and other criteria. These recommendations are based on expert assessment in which the level of evidence is based on available information using standardized criteria. Experts agree that before systemic treatment is initiated it is important to resolve bile and duodenal obstruction by endoscopic stent placement as well as to have adequate pain control and nutritional status.

ESMO Guidelines recommend that patients with advanced PDAC and ECOG 0 or 1 should receive first-line treatment with either the combination of gemcitabine (GEM) and nab-paclitaxel or FOLFIRINOX [10]. As discussed below, both of these regimens have shown improvement in overall survival (OS) in randomized phase 3 studies (IA). Patients with ECOG 2 and/or bilirubin levels $>1.5$ upper normal limit (UNL) should be treated with single agent GEM (IA) [14]. However, the combination of nab-paclitaxel and GEM is recommended for patients in whom the ECOG 2 is a consequence of high tumor burden (IIB). The role of other combinations based on GEM, including GEM and erlotinib, is less clear due to the clinical significance in increase in median survival of only 14 days $[15,16]$. For patients with ECOG 3 and 4 the recommended treatment is palliative care. For patients with DNA damage repair deficits (BRCA mutations) or acinar type tumors, due to a higher probability of response rate, the recommended treatment is platinum analogs [10].

Table 1. ESMO, NCCN, ASCO AND SEOM guidelines [10-13].

\begin{tabular}{|c|c|c|c|c|}
\hline GUIDELINES & ECOG 0-1 & ECOG 2 & ECOG 3-4 & OTHERS \\
\hline ESMO [10] & $\begin{array}{l}\text { Nab-paclitaxel + GEM (IA) } \\
\text { or } \\
\text { FOLFIRINOX (IA) }\end{array}$ & $\begin{array}{l}\text { ECOG } 2 \text { or bilirubin levels }>1.5 \text { UNL: } \\
\text { GEM (IA) } \\
\text { ECOG } 2 \text { as consequence of high tumor } \\
\text { burden: } \\
\text { Nab-paclitaxel + GEM (IIB) }\end{array}$ & Palliative Care & $\begin{array}{l}\text { BRCA mutations o acinar } \\
\text { type tumor: } \\
\text { Platinum salt treatment or } \\
\text { platinum analogs } \\
\text { combinations }\end{array}$ \\
\hline NCCN [12] & $\begin{array}{l}\text { Combination of nab-paclitaxel + GEM } \\
\text { (category } 1 \text { ) } \\
\text { or } \\
\text { FOLFIRINOX (category 1) } \\
\text { or } \\
\text { Other options }{ }^{* *}\end{array}$ & $\begin{array}{l}\text { GEM (category } 1 \text { ) } \\
\text { KPS } \geq 70 \text { : } \\
\text { Combination of nab-paclitaxel + GEM ( } 2 \mathrm{~A})\end{array}$ & $\begin{array}{l}\text { Poor performance status: } \\
\text { GEM (category 1) } \\
\text { or } \\
\text { Palliative Care }\end{array}$ & \\
\hline ASCO [13] & $\begin{array}{l}\text { ECOG 0-1 and: } \\
\text { Favorable comorbidity profile: } \\
\text { FOLFIRINOX (Type: evidence based, } \\
\text { benefits outweigh harms; Evidence quality: } \\
\text { intermediate; Strength of recommendation: } \\
\text { strong) } \\
\text { Relatively favorable comorbidity: } \\
\text { Combination of nab-paclitaxel + GEM (Type: } \\
\text { evidence based, benefits outweigh harms; } \\
\text { Evidence quality: intermediate; Strength of } \\
\text { recommendation: strong) }\end{array}$ & $\begin{array}{l}\text { ECOG } 2 \text { or a comorbidity profile that } \\
\text { precludes more-aggressive regimens and } \\
\text { who wish to pursue cancer-directed } \\
\text { therapy: } \\
\text { GEM or GEM + erlotinib or capecitabine } \\
\text { (Type: evidence based, benefits outweigh } \\
\text { harms; Evidence quality: intermediate; } \\
\text { Strength of recommendation: moderate) }\end{array}$ & $\begin{array}{l}\text { ECOG } \geq 3 \text { or with poorly controlled } \\
\text { comorbid conditions despite } \\
\text { ongoing active medical care: } \\
\text { They should be offered } \\
\text { cancer-directed therapy only on a } \\
\text { case-by-case basis. The major } \\
\text { emphasis should be on optimizing } \\
\text { supportive care measures (Type: } \\
\text { evidence based, benefits outweigh } \\
\text { harms; Evidence quality: } \\
\text { intermediate; } \\
\text { Strength of recommendation: } \\
\text { moderate). }\end{array}$ & \\
\hline SEOM [11] & $\begin{array}{l}\text { and }<75 \text { and fit: } \\
\text { Combination of nab-paclitaxel + GEM (IA) or } \\
\text { FOLFIRINOX (IA) }\end{array}$ & $\begin{array}{l}\text { or fit > } 75 \text { years: } \\
\text { Combination of nab-paclitaxel + GEM (IIB) } \\
\text { or } \\
\text { GEM (IB) }\end{array}$ & $\begin{array}{l}\text { or frail patients: } \\
\text { Best supportive care }\end{array}$ & $\begin{array}{l}B R C A \text { or } P A L B 2 \text { mutations: } \\
\text { Platinum-based treatments }\end{array}$ \\
\hline
\end{tabular}

*GEM: Gemcitabine; KPS: Karnofsky performance status; ** Other options: GEM + erlotinib (category 1); GEM-based combination therapy; GEM (category 1); Capecitabine or continuous infusion 5FU (category 2B); fluoropyrimidine + oxaliplatin (category 2B) 
The NCCN guidelines recommend that patients with ECOG 0-1 participate in a clinical trial and if not feasible, either FOLFIRINOX or the combination of nab-paclitaxel + GEM (category 1) as preferred options but it also include other GEM-based combinations including GEM and erlotinib as category 1 , although with a very limited benefit [12]. The combination of nab-paclitaxel + GEM is a reasonable treatment option also for patients with Karnofsky performance status $\geq 70$. For patients with poor performance status (PS), the recommended treatment is either GEM (category 1) or palliative care.

The ASCO guidelines further recommend FOLFIRINOX for patients with a favorable comorbidity profile who prefer aggressive treatment and have the support system for such aggressive medical therapy while the combination of nab-paclitaxel + GEM is recommended for patients with relatively favorable comorbidity profile who prefer and have the support system for relatively less aggressive medical therapy [13]. The combination of nab-paclitaxel + GEM is less strict with regards to hematological parameters, age and PS requirements, compared to FOLFIRINOX.

Table 2. Algorithm for first line treatment for mPDAC, based on patients' characteristics [18].

\begin{tabular}{|c|c|}
\hline Patient's characteristics & $\begin{array}{l}\text { First line treatment } \\
\text { recommendations }\end{array}$ \\
\hline $\begin{array}{l}\text { Candidates suitable for chemotherapy treatment } \\
\text { WITHOUT limitations: } \\
\text { ALL ítems should be met: } \\
\text { * ECOG } 0-1 \\
\text { * Age } \leq 75 \text { years } \\
\text { * Bilirubin } \leq 1.5 \text { UNL } \\
\text { * Good nutritional status } \\
\text { * Lack of comorbidities }\end{array}$ & $\begin{array}{l}\text { or } \\
\text { FOLFIRINOX (IA) }\end{array}$ \\
\hline $\begin{array}{l}\text { Candidates suitable for chemotherapy WITH } \\
\text { limitations: } \\
\text { At least ONE of the ítems should be met: } \\
{ }^{*} \text { ECOG } 2 \\
\text { * Age > } 75 \text { years } \\
\text { * Mild to moderate organ dysfunction: } \\
\text { - Neurological, endocrine-metabolic, renal } \\
\text { - Non-obstructive reversible (dose adjustment) } \\
\text { liver dysfunction (hyperbilirubinemia): } \\
\text { > Non tumor induced (prior liver disease): } \\
\text { Bilirubin levels: } 1.5 \text { - } 3 \mathrm{mg} / \mathrm{dL} \\
\text { > Tumor induced (obstructive jaundice or liver } \\
\text { infiltration): Bilirubin levels: } 1.5 \text { - } \leq 5 \mathrm{mg} / \mathrm{dL} \\
\text { - Cardiac dysfunction } \\
\text { * Recent VTD } \\
\text { * Malnutrition/tumor cachexia }\end{array}$ & $\begin{array}{l}\text { ECOG 2: } \\
\text { Due to tumor burden: } \\
\text { Nab-paclitaxel + GEM } \\
\text { Due to comorbitides: } \\
\text { Nab-paclitaxel + GEM or GEM }\end{array}$ \\
\hline $\begin{array}{l}\text { Candidates NOT suitable for chemotherapy: } \\
\text { The presence of AT LEAST ONE of the following } \\
\text { factors: } \\
\text { * ECOG } 3-4 \\
\text { * Severe organ dysfunction } \\
\text { - Neurological, endocrine-metabolic, renal... } \\
\text { - Liver dysfunction (hyperbilirubinemia): } \\
\text { > Non tumor-induced (prior liver disease): > } 3 \\
\text { mg/dL } \\
\text { > Tumor-induced (obstructive jaundice or liver } \\
\text { infiltration): }>5 \mathrm{mg} / \mathrm{dL}\end{array}$ & $\begin{array}{l}\text { * Support treatment in } \\
\text { Palliative Care Unit }\end{array}$ \\
\hline
\end{tabular}

SEOM guidelines also incorporate age and fitness in the decision making algorithm [11]. Thus, patients with ECOG $0-1$, age $<75$ years and fit can be treated with either FOLFIRINOX or the combination of nab-paclitaxel + GEM. Patients with ECOG 2 or those who are fit but age $>75$ years should be treated with the combination of nab-paclitaxel + GEM or GEM alone. For patients with ECOG 3-4 or frail the recommended approach is best supportive care [11]. Platinum based treatments are recommended for patients with BRCA or PALB2 mutations [17].

In addition, a recent Spanish consensus has also reviewed the different therapeutic options in mPDAC based on patient's characteristics [18]. As shown in Table 2, for those patients that are candidates for chemotherapy treatment with no limitations, treatment options include nab-paclitaxel + GEM or FOLFIRINOX. However, for those patients that are candidates for chemotherapy treatment with limitations, treatment options include the combination of nab-paclitaxel + GEM (ECOG 2 due to tumor burden) or either the combination of nab-paclitaxel + GEM or GEM monotherapy (ECOG 2 due to comorbidities). Finally, for those patients that are not candidates for chemotherapy treatment, the only option is support treatment in Palliate Care Units [18].

\section{MPACT vs PRODIGE in routine clinical practice}

As mentioned above and for the most part, systemic treatment of patients with mPDAC is currently based on the results of two randomized clinical trials that compared first-line FOLFIRINOX or the combination of nab-paclitaxel + GEM versus GEM and demonstrated a statistically significant improvement in median OS, the primary aim of both studies $[6,7]$. As summarized in Table 3, median OS of patients treated with FOLFIRINOX compared to GEM in the PRODIGE study was 11.1 months vs. 6.8 months, respectively [7]. The combination of nabpaclitaxel + GEM resulted in an improvement in median OS (from 6.7 months to 8.7 months) compared to GEM [6]. However, these two regimens (the combination of nab-paclitaxel + GEM and FOLFI RINOX) have not been compared head to head and therefore, it is not known which one, if any, is better.

In routine clinical practice the key issue is to select, from available options, the regimen that better suits individual patients. In making this selection, a number of factors need to be considered. The clinical trial population is indeed a patient sample from the overall patient population and the goal is to apply the results from the sample to the population. Factors to consider include ECOG PS, age, disease extent, comorbidities, nutritional status, patient's preferences, family support, efficacy of the regimen, 
tolerability, treatment sequence, and finally treatment goals.

Table 3. MPACT and PRODIGE studies: Comparison of study characteristics, demographics, outcome, safety and treatment administration $[6,7]$.

\begin{tabular}{|c|c|c|}
\hline & MPACT & PRODIGE \\
\hline & \multicolumn{2}{|c|}{ Study Characteristics } \\
\hline Study design & Phase III & Phase III \\
\hline Number of patients & 861 & 342 \\
\hline Continents & 3 & 1 \\
\hline Countries & 11 & 1 \\
\hline Number of centers & 151 & 48 \\
\hline Statistical Power & $90 \%$ & $80 \%$ \\
\hline \multirow[t]{2}{*}{ Centralization } & Yes & No \\
\hline & \multicolumn{2}{|c|}{ Demographic Characteristics } \\
\hline Age (range) & $62(27-86)$ & $61(25-76)$ \\
\hline \multicolumn{3}{|l|}{ ECOG } \\
\hline 0 & $16 \%$ & $37 \%$ \\
\hline 2 & $8 \%$ & $1 \%$ \\
\hline Head of Pancreas & $44 \%$ & $39 \%$ \\
\hline Biliary Stent & $\begin{array}{l}19 \% \\
\text { (no limitation) }\end{array}$ & $\begin{array}{l}16 \% \\
\text { (preferably not) }\end{array}$ \\
\hline Median Metastatic Sites & 3 or + & 2 \\
\hline \multirow[t]{2}{*}{ CA $19.9>59 \times$ UNL } & $52 \%$ & $41.5 \%$ \\
\hline & \multicolumn{2}{|l|}{ Outcome } \\
\hline OS (median, months) & 8.7 & 11.1 \\
\hline \multicolumn{3}{|l|}{$\%$ patients alive } \\
\hline 12 & 35 & 48.4 \\
\hline 18 & 16 & 18.6 \\
\hline 24 & 10 & NR \\
\hline 42 & 2 & NR \\
\hline \multicolumn{3}{|l|}{ Response Rate (\%) } \\
\hline Investigator assessment & 29 & 31.6 \\
\hline Central review & 23 & NR \\
\hline
\end{tabular}

Table 4. MPACT trial: Median survival in different populations [21,24].

\begin{tabular}{ll}
\hline Populations & \\
\hline & MPACT median survival \\
Population $<65$ years & $\mathbf{9 . 6}$ months \\
KPS of $90-100 \%$ & 9.7 months \\
KPS of $100 \%$ & 12.6 months \\
Western European countries & 10.7 months \\
& YOSEMITE median survival \\
ECOG 0-1 & Not reached in the control group \\
& $(>13.2$ months in the experimental arm)
\end{tabular}

When comparing the design and population enrolled in both studies there are important differences that can affect the results achieved. Table 3 provides a comparative summary of study design, patients' characteristics and outcome of the studies. Multiple factors, such as age and PS, can affect outcome: For example, in the MPACT trial, the median survival of patients with KPS of $90-100 \%$ was 9.7 months [19]. In addition, the median survival with KPS $100 \%$ was 12.6 months. Likewise, patients with only one metastatic site survived 13.5 months [20]. An analysis of survival in patients from Western European countries showed 10.7 month median survival [21]. Finally, in the population $<65$ years old the median survival was 9.6 months (Table 4). While the studies are similar with regards to median age, gender, stage of disease and liver involvement, the percentages of elderly patients, those with high tumor burden, and those with ECOG 2 were higher in the MPACT trial. Hence, while the results with MPACT are inferior numerically, patients' status was worse, and the results therefore are more generalizable and convenient. Recent practice patterns analysis in the US shows that the combination of nab-paclitaxel + GEM is progressively becoming the most frequently used regimen [22].

Both regimens resulted in improvement in quality of life. In the PRODIGE study, patients treated with FOLFIRINOX had a significantly lower rate of deterioration [23]. Likewise, patients with good performance status treated with the combination of nab-paclitaxel + GEM in the MPACT trial were more likely to maintain a high functional status as compared to those treated with GEM alone [24]. The combination of nab-paclitaxel + GEM treatment resulted in a $20.7 \%$ improvement in quality of life adjusted survival in mPDAC patients [25]. In addition, as discussed below, the combination of nab-paclitaxel + GEM was better tolerated in terms of toxicity (Table 5).

Table 5. MPACT and PRODIGE studies: Most significant toxicities $[3,4]$.

\begin{tabular}{|c|c|c|}
\hline & MPACT & PRODIGE \\
\hline Fatigue & $17 \%$ & $23.6 \%$ \\
\hline Diarrhea & $6 \%$ & $12.7 \%$ \\
\hline Vomiting & - & $14.5 \%$ \\
\hline Neuropathy & $17 \% * *$ & $9 \%$ \\
\hline Ischemic cardiopathy & No limitations & Limitations (probably) \\
\hline Biliary prothesis & No limitations $(19 \%)$ & Preferably no biliary stent $(15.8 \%)$ \\
\hline VTE & No limitations & Increases VTE risk (PTE: 6.6\%) \\
\hline Anemia & $13 \%$ & $7.8 \%$ \\
\hline Thrombopenia & $13 \%$ & $9 \%$ \\
\hline Febrile neutropenia & $3 \%$ & $5.4 \%$ \\
\hline \multicolumn{3}{|c|}{$\begin{array}{l}{ }^{*} \text { VTE: venous thromboembolic events; PTE: Pulmonary thromboembolism } \\
\text { ** In the GEM + nab-paclitaxel group, the median time to the first occurrence of } \\
\text { grade } 3 \text { neuropathy was } 140 \text { days, and the median time to improvement from grade } \\
3 \text { to grade } 2 \text { was } 21 \text { days and to grade } 1 \text { or resolution of the event was } 29 \text { days. Of } \\
\text { the patients who had grade } 3 \text { peripheral neuropathy, } 44 \% \text { resumed treatment at a } \\
\text { reduced dose of nab-paclitaxel within a median of } 23 \text { days after the onset of a grade } \\
3 \text { event. }\end{array}$} \\
\hline
\end{tabular}

Recently, several retrospective studies have analyzed the toxicity and efficacy of these regimens in daily clinical practice. A study that analyzed the median survival of patients treated with GEM based on their eligibility to receive either FOLFIRINOX or the combination of nab-paclitaxel + GEM showed that eligible patients had better survival than those that were not [26]. Thus, candidates to receive FOLFIRINOX had a median survival of 8.6 months vs. 4.7 months for those that were not fit to receive FOLFIRINOX $(p<0.001)$. Likewise, OS for candidates to receive the combination of nab-paclitaxel + GEM was 6.7 months compared to 4.9 months for those who 
were ineligible $(p=0.008)$. Overall, double number of patients $(45.2 \%)$, were potentially eligible for the combination of nab-paclitaxel + GEM vs. for FOLFIRINOX (24.7\%). Poor ECOG (PS $\geq 2)$ and age (> 76 years old) were the main limiting factors for FOLFIRINOX eligibility [27]. Furthermore, in an analysis of 2,422 patients with advanced PDAC treated in routine clinical practice in the US, the median OS for FOLFIRINOX treated patients was 11.2 months vs. 10.2 for the combination of nab-paclitaxel + GEM [27]. Time to treatment discontinuation was similar in patients treated with FOLFIRINOX vs. the combination of nab-paclitaxel + GEM in community oncology settings while the overall incidence of grade $3 / 4$ toxicity was higher ( $95 \%$ vs. $84 \%$ ), in particular diarrhea (15\% vs. $9 \%)$, and dehydration $(21 \%$ vs. $14 \%)$ [28]. Thirty nine percent of FOLFIRINOX treated patients vs. $8 \%$ of patients treated with the combination of nab-paclitaxel + GEM required G-CSF support [28]. In addition to the results of retrospective studies and analysis of routine clinical practice, another evidence to support the efficacy of nab-paclitaxel + GEM comes from the analysis of recent randomized clinical trials in which the regimen was selected as the control arm. For example, the Yosemite study, recently presented, compared nab-paclitaxel and GEM with combinations of this regimen with demcizumab, an inhibitor of the DLL4 notch ligand. The overall results of the study were negative and the combination treatment did not improve the outcome of the patients. While the reasons for this negative results are not known, an interesting observation is that in this highly selected group of patients, the combination of nab-paclitaxel and GEM showed very encouraging results with an overall response rate of $41.2 \%, 70.6 \%$ overall clinical benefit, and PFS of 5.49 months. OS, that was 13.24 months for the experimental arm, had not been reached in the control arm at the time of the last update. A number of randomized studies in highly selected patient populations using nab-paclitaxel and GEM as the control arm are now being conducted and their results, that will be presented in the near future, will provide additional support of the role of nab-paclitaxel and GEM in highly selected patients with advanced PDAC [29].

Interestingly, elderly patients treated with the combination of nab-paclitaxel + GEM or who received the combination of nab-paclitaxel + GEM in routine clinical setting do not have worse outcome in retrospective studies but develop more toxicity including hematological toxicity, neurotoxicity, and fatigue [30,31]. No differences in survival were observed in patients with ECOG 2 or bile stents either [32].
FOLFIRINOX regimen has shown to have similar efficacy in patients $>70$ years compared to younger patients in small studies, achieving OS of 11.6 and 11 months, respectively [33,34]. However, as mentioned above, and discussed below, FOLFIRINOX is associated with substantial grade 3 and 4 toxicity events. For that reason, the practical application of the published regimen is not clear. In a retrospective study of 224 patients treated with FOLFIRINOX, less than half of the patients received the full dose, almost half of the patients did not receive the 5 -fluorouracil (5FU) bolus and $25 \%$ received $<70 \%$ of the full dose. In addition, $40 \%$ required G-CSF support [35].

Recent studies with modified schemas of FOLFIRINOX have shown good results with regards to OS [36-38]. The results of a retrospective study that analyzed the outcome of 137 PDAC patients treated with modified FOLFIRINOX show that those who had two or more risk factors (liver metastases, PS1 and lymphocyte ratio $>4$ ) had a significantly lower OS (7.4 months) compared to those with one or none risk factors (11.1 vs. 17.6 months, respectively) [39]. Another retrospective study analyzed the use of FOLFIRINOX in daily clinical practice in 292 PDAC patients in Italy [40]. The results showed that $70 \%$ of the patients had ECOG $0,9 \%$ had stage I-II and $30 \%$ of the patients had locally advanced disease. With regards to OS, those patients treated with the modified schema had a significantly longer OS compared to regular FOLFIRINOX (18 vs. 12 months) [40].

In another retrospective study, conducted on Japanese population, that compared a modified FOLFIRINOX regimen vs nab-paclitaxel + GEM in 135 patients with similar baseline characteristics showed that nab-paclitaxel + GEM resulted in higher response rate $(39 \%$ vs. $27 \%)$, median OS (14 months vs. 11.5 months) and one-year survival (67\% vs. $44 \%)$, and a similar toxicity profile [41].

The impact of dose modification on the combination of nab-paclitaxel + GEM has been less studied. However, the limited evidence available suggests that tolerability is similar, regardless of age, PS, as well as the presence of biliary stent. Thus, a phase 2 study of 47 patients treated with full dose of the combination of nab-paclitaxel + GEM in a biweekly schedule reported an overall median survival of 11.1 months, with only $10 \%$ grade $3-4$ neutropenia and $6 \%$ grade 3-4 fatigue [42].

The exploratory analysis conducted by Scheithauer et al. in the MPACT trial suggested dose reductions and delays were effective when necessary to ameliorate toxicity allowing greater treatment exposure without compromising efficacy [43]. In addition, patients with biliary stent treated with the 
combination of nab-paclitaxel + GEM appeared to have same outcome in retrospective studies [44].

One important clinical question is the most appropriate regimen for patients who have received adjuvant treatment with GEM after resection of a localized tumor and subsequently develop disease progression. An analysis of 90 of such patients who received the combination of nab-paclitaxel + GEM after relapse showed a median OS of 15 months. Survival changed with the disease free interval (DFI) and increased from 8.7 months in patients DFI $<6$ months to 16.3 months in those with DFI $>$ than 12 months [45].

\section{Tolerability}

An important consideration when selecting a treatment regimen is the expected frequency and severity of side effects. The occurrence of side effects is related to the patient baseline characteristics and comorbidities such as age, ECOG, and bile duct stent among others. In addition, it is also crucial to gauge the impact that any given toxicity could have in the patient overall health status; appropriate preventive measures; access to care should an event occur as well as to provide a concrete and well detailed plan on how to manage events should they occur. Table 5 provides numerical information of the most relevant toxicity events reported with these regimens [6,7]. In this section venous thromboembolic events (VTE), cardiac toxicities and gastrointestinal complications will be reviewed.

The French physician Jean-Baptiste Bouillaud reported the association between cancer and VTE in 1823 [46]. It has been shown that cancer patients have a 4 to 7-fold increment in the risk of VTE. The prevalence of arterial ischemic events ranges from $1.5 \%$ to $3.1 \%$, while VTE events show a 28 -fold increase risk [47-49]. Cancer-related VTE accounts for increased mortality and morbidity: mortality after a discharge for VTE event is nearly doubled and the risk of death increases up to eightfold after an acute VTE in patients with cancer as compared with non-cancer patients. In addition, patients with cancer-related thrombosis have a lower survival rate than cancer patients without thrombosis [50].

In 2016, international clinical practice guidelines were released by an international consensus-working group that pooled the existing data with the intent of making the Good Clinical Practice Guidelines (GCPG) easier to use at each national level [51].

FOLFIRINOX increases the risk of VTE due to several factors including the drugs themselves (irinotecan per se and in combination with 5FU and LV); as well as due to the need to use a central line and frequent use of G-CSF [52,53]. An analysis of risk factors for VTE in 570 active cancer-associated incident VTE (cases) and 604 matched controls with active cancer identified as independent risk factors the following variables: cancer site, disease stage, liver metastasis, progesterone, being underweight or obese, hospitalization or nursing home, central venous catheter and infection [54].

Pancreas cancer diagnoses conferred the highest risk [55]. Chemotherapy treatment is associated with a 1.8-fold increase risk of VTE [52]. In a study at Memorial Sloan Kettering Cancer Center conducted from 2003 to 2005, 115 of 2,120 cancer patients being treated for advanced malignancies develop VTE [52]. The tumor location (gastrointestinal tumors) and irinotecan treatment were independent risk factors for VTE. Patients with PDAC had a 2.26 (1.06 - 4.80) hazard ratio (HR) of developing VTE. Likewise, treatment with irinotecan increased the HR to develop a VTE to 1.89 (1.29 - 3.59). Indeed, in the colorectal cancer randomized trials CALGB89803 and N9741, irinotecan treatment combined with $5 \mathrm{FU}$ and $\mathrm{LV}$ was associated with a $2.4 \%$ and $3.1 \%$ of patients experiencing treatment related death respectively [56]. In these studies, $0.8 \%$ and $1 \%$ of deaths were either induced or exacerbated by vascular events.

Other risk factors for VTE include the need of a central venous catheter and use of G-CSF in up to half of the patients $[53,57,58]$. Studies have shown that there is a $0.3 \%$ to $28 \%$ incidence of catheter related VTE in patients with cancer [57]. In addition, the use of G-CSF increases the risk of VTE by 1.69 (range: 1.09-2.64) [54]. With regards to the combination of nab-paclitaxel + GEM, its use has not been associated with a significant increase in the risk of VTE [6]. However, the results of the PROTECHT study have shown a significant increase in VTE risk associated to GEM [59].

With regards to cardiotoxicity, it has been long recognized than 5FU causes cardiac toxicity [60]. However, the syndrome of 5FU -associated cardiotoxicity remains poorly defined and its incidence may be underestimated. Large series suggest that clinically demonstrated 5FU-related cardiotoxicity ranges from 1.6 to $4 \%$ [60].

Importantly, the presence of prior cardiac history is not predictive as only $14 \%$ of patients have a history of heart disease and cardiac risk factors are present in only $37 \%$ of patients. However, the probability of EKG changes in 10 fold higher in patients with prior history of cardiac diseases (15 vs. $1.5 \%, p<0.001$ ) [61]. Cardiac events appear to be independent of dose and are higher in patients treated with continuous infusion as compared to bolus regimens ( 12.5 vs. $3.5 \%$, $p=0.024)$ [62]. 
Previous studies also suggest that the addition of $\mathrm{LV}$ to continuous infusion of 5FU results in a further increment of cardiotoxicity [60]. The pathophysiology of 5FU cardiac toxicity is not fully understood, and different alteration, such as coronary artery thrombosis, arteritis, or vasospasm have been proposed as underlying mechanisms. Direct toxicity to the myocardium and autoimmune phenomena have also been implicated. Clinically, most cases consist of ischemic events (angina myocardial infarction), and arrhythmias. Most frequent EKG changes include ischemia or ST-T segment elevation. Approximately $12 \%$ of the patients develop enzymatic changes, with a reported mortality rate of $8 \%$ [60].

With regards to the combination of nab-paclitaxel + GEM, less ischemic events have been reported, compared to FOLFIRINOX regimen. As mentioned before, this is related to the higher cardiotoxicity associated to 5FU [63-65].

Pancreatic exocrine insufficiency (PEI) is a known complication of both benign and malignant pancreatic diseases, pancreatic resection, and postsurgical alteration of the anatomy of the foregut. It is defined as inadequate pancreatic enzyme activity caused by insufficient pancreatic enzyme production/ activation, or altered enzyme deactivation. The physiologic biphasic pattern of pancreatic enzyme release is absent in patients with mPDAC [66].

Unfortunately, PEI is often overlooked in cancer patients, as the focus of attention is their baseline disease. However, PEI occurs frequently in PDAC affecting both patients with and without surgical resection [67]. A prospective trial conducted in 194 mPDAC patients found that $25 \%$ of the patients presented extreme levels PEI (levels of fecal elastase-1 $<25 \mu \mathrm{g} / \mathrm{g}$ ), while $14 \%$ and $11 \%$ presented severe or moderate PEI [68]. The most frequent tumor location in patients with extreme PEI was the head of the pancreas. In addition, extreme PEI was significantly associated with jaundice and a with worse prognosis (median OS of 7 months vs. 11 months, $p=0.031$ ) [68]. Given this high prevalence, physicians should focus on diagnosing and treating PEI and to optimize nutritional status and physical condition, especially for those patients undergoing palliative chemotherapy. These issues, that are innate to mPDAC, are further exacerbated in patients treated with FOLFIRINOX because of the high incidence of grade 3 vomiting (14.5\%) and diarrhea (12.7\%) as compared to the combination of nab-paclitaxel and GEM (no vomiting and diarrhea $6 \%$ ) [6,7].

mPDAC patients often have a decreased nutritional intake secondary to a variety of factors, including anorexia, early satiety, nausea, pain, anxiety, and depression. Eighty percent of mPDAC patients have weight loss at presentation. mPDAC patients often report a loss of approximately $15 \%$ of their pre-illness weight by the time of diagnosis, and the weight loss continues with a median loss by the time of death of $25 \%$ of pre-illness weight [69].

\section{Sequential treatment}

The availability of more effective first line treatments (the combination of nab-paclitaxel + GEM and FOLFIRINOX based on the results of phase III studies) is allowing the development of second line treatment options based on the results of phase II and phase III studies for patients with mPDAC [6-9, 70-72].

Thus, the results of the CONKO-003 study, that analyzed the efficacy of second line oxaliplatin and $5 \mathrm{FU} / \mathrm{LV}$ versus 5FU/LV alone for GEM refractory pancreatic cancer, showed that the combination significantly extended the duration of OS when compared to 5FU/LV alone in patients with advanced GEM refractory pancreatic cancer [9].

On the other hand, the PANCREOX study, that compared infusional 5FU/LV vs. mFOLFOX6 (infusional 5FU/LV and oxaliplatin) did not demonstrate differences in PFS or OS between these two regimens, questioning the role of the addition of oxaliplatin for the management of patients previously treated with GEM-based-regimens [73].

Finally, the results of the NAPOLI phase III study, that analyzed the effect of nanoliposomal irinotecan (NalIRI) alone or combined with 5FU/LV in a phase 3 trial in patients with metastatic PDAC previously treated with GEM-based therapies showed that extends survival (6.1 vs 4.2 months), with a manageable safety profile [8].

Indeed, patients who receive three lines of treatment are not rare these days. Similar to other diseases like colon cancer for example, the availability of second and even third line treatments imply that the management of the disease should be viewed as a continuum of care of several lines of treatment rather than compartmentalized treatments. However, there are no clear guidelines regarding the optimal sequence of treatment in metastatic PDAC, in part due to the lack of head to head comparison between possible approaches. Factors to consider in making these decisions include the ECOG performance status, comorbidities, residual toxicities (i.e. neuropathy), prior treatments, and patient's goals and preferences. Algorithms for sequential management of patients with PDAC, based on expert opinions are emerging. Table 6 provides a potential treatment sequencing approach for PDAC based on the review of the available literature. 
Table 6. Potential treatment sequencing approach for mPDAC.

\begin{tabular}{lll}
\hline First line & Second line & Third line \\
\hline Nab-paclitaxel + GEM & 5FU based regimen: & Platinum-based regimen \\
& 5FU/LV & or \\
& NalIRI + 5FU/LV & NalIRI \\
& Oxaliplatin + 5FU/LV & \\
Capecitabine & \\
FOLFIRINOX & GEM based-regimen & $?$
\end{tabular}

${ }^{*}$ GEM: Gemcitabine; 5FU: 5-fluorouracil; LV: Leucovorin; NalIRI: liposomal irinotecan

Table 7. mPDAC: Sequential treatments: Retrospective data [74-78].

\begin{tabular}{llll}
\hline Study & Treatment Strategy & N & $\begin{array}{l}\text { OS (median, } \\
\text { months) }\end{array}$ \\
\hline MPACT [78] & Nab-paclitaxel + GEM, 5FU/Capecitabine & 132 & 13.5 \\
& Nab-paclitaxel + GEM -FOLFIRINOX & 18 & 15.7 \\
& Nab-paclitaxel + GEM -FOLFOX & 36 & 13.7 \\
Gilabert et al & GEM-based followed Ox-based & 16 & 14.9 \\
[74] & & & \\
Giordano et al & Nab-paclitaxel + GEM -second Line & 102 & 13.5 \\
[75] & chemotherapy & 56 & 12.8 \\
& Nab-paclitaxel + GEM -FOLFOL/XELOX & 24 & 13.2 \\
& Nab-paclitaxel + GEM -FOLFIRI & 22 & 13.8 \\
Braiteh et al & Nab-paclitaxel + GEM -FOLFIRINOX & & \\
[76] & Nab-paclitaxel + GEM -5FU based & 26 & 12.7 \\
& regimen & 41 & 8.4 \\
Schimdt et al & FOLFIRINOX-GEM based regimen & & \\
[77] & FOLFIRINOX- Nab-paclitaxel + GEM & 20 & 10.8 \\
& Nab-paclitaxel + GEM -FOLFIRINOX & 6 & 18 \\
& FOLFIRINOX/FOLFOX- Nab-paclitaxel + & 127 & 12.1 \\
& GEM & 10 & 12.2 \\
& Nab-paclitaxel + GEM & & \\
& -FOLFIRINOX/FOLFOX & & \\
\hline
\end{tabular}

*GEM: Gemcitabine; OX: Oxaliplati

Randomized clinical trials in second line setting are only available for patients who received GEM based chemotherapy in the first line setting (Tables 7 and 8$)[7,74-77]$. The vast majority of these patients, however, did not receive the combination of nab-paclitaxel + GEM [8,9,71,72]. Notwithstanding differences in study design and the small samples sizes of the trials, there are conflicting results for FOLFOX-like regimens with one positive and another negative trial. The most consistent data currently available is for liposomal irinotecan (NalIRI) + $5 \mathrm{FU} / \mathrm{LV}$ [8]. It should be noted, however, very few of these patients received the combination of nab-paclitaxel + GEM in the first line setting, the preferred GEM combination used these days. Likewise, there is no randomized trial of second line treatment after first line treatment with FOLFIRINOX.

In the lack of randomized trials in the second line setting after the combination of nab-paclitaxel + GEM as first line, an approach to determine the results with second line treatments is to analyze the outcome of patients who received second line treatment after first line with the combination of nab-paclitaxel + GEM in the MPACT trial [78]. A total of 347, approximately $40 \%$ of patients from the MPACT trial received second line treatment. More than $60 \%$ of the patients who received a second line treatment were KPS 90-100\% and the most common regimen used (78\%) was $5 \mathrm{FU}$ based. Regardless of the second line treatment received, OS was longer for patients who had received the combination of nab-paclitaxel + GEM as compared to GEM alone (12.8 vs. 9.9 months, $p=$ 0.015 ) in the first line setting; however, no differences in survival were noted for second line treatment based on first line results. The best OS as obtained in patients who received first line with the combination of nab-paclitaxel + GEM followed by FOLFIRINOX (15.7 months). These results are consistent with a retrospective analysis that compared the outcome of patients receiving two or more lines of chemotherapy in order to define the optimal sequence of treatments [74]. Patients were classified in two groups based on the first line treatment they had received (5FU-based, 59 patients) or GEM-based (31 patients). OS was significantly longer in patients who had received gemcitabine-based first line treatment (14.9 months vs. 13.8 months, $p=0.046)$. Similar results have been reported in other studies indicating that patients who receive second line treatment, as expected, have better survival and that results appear to be superior with the sequence of gemcitabine-based versus $5 \mathrm{FU}$ based in the first line. In the PRODIGE study a total of 165 patients $(48.2 \%)$ of the patients received second line treatment (80 patients in the FOLFIRINOX group and in 85 patients in the GEM group) [7]. No difference in median survival was noted between the groups (4.4 months in each group) from the introduction of second-line therapy. The most common second line regimens were as follows: in the FOLFIRINOX group, GEM (in $82.5 \%$ of the patients) or a GEM-based combination (in 12.5\%), and in the gemcitabine group, a combination of 5FU, LV, and oxaliplatin (FOLFOX) (in 49.4\%); GEM plus oxaliplatin (in 17.6\%); a regimen of 5FU and LV plus cisplatin every 2 weeks (in 16.5\%); and FOLFIRINOX (in 4.7\%). It is also important to mention the results a small study $(n=57)$ conducted in France (AGEO study) that analysed the efficacy of nab-paclitaxel + GEM in metastatic PDAC after FOLFIRINOX failure [79].

Table 8. Second line treatment: Results of randomized studies $[8,9,71,72]$.

\begin{tabular}{|c|c|c|c|c|c|c|}
\hline Reference & Study design & Number of patients & First line & Second line & PFS (median, months) & OS (median, months) \\
\hline CONKO-003 [9] & Phase III & 168 & GEM & OFF vs 5FU/LV & 2.9 vs 2.0 & 5.9 vs 3.3 \\
\hline PANCREOX [72] & Phase III & 108 & GEM based & FOLFOX vs 5FU/LV & 3.1 vs 2.9 & 6.1 vs 9.9 \\
\hline NAPOLI-1 [8] & Phase III & 417 & GEM based & NalIRI +/- 5FU/LV & 3.1 vs 1.5 & 6.1 vs 4.2 \\
\hline SWOG S115 [71] & Phase II & 115 & GEM & FOLFOX vs MK2006 + selumetinib & 2.0 vs 1.5 & 6.9 vs 4.0 \\
\hline
\end{tabular}

*GEM: Gemcitabine; 5FU: 5-fluorouracil; LV: Leucovorin; OFF: Oxaliplatin/5FU/LV; NalIRI: liposomal irinotecan 
Disease control rate and objective response rate were $58 \%$ and $17.5 \%$ respectively. In addition, median OS and median PFS were 8.8 and 5.1 months. The reported median OS survival since the commencement of the first-line chemotherapy was 18 months. The results suggest that the administration of the combination seems to be effective, with a manageable toxicity profile, after FOLFIRINOX failure.

Treatment guidelines recommendations by ESMO, NCCN and SEOM indicate that second line treatment should be considered for patients with good performance status [10-12]. For patients who have received gemcitabine-based chemotherapy in the first line setting, the NCCN Guidelines recommend either 5FU + NalIRI (IA) or other fluoropyrimidine based treatment [9]. ESMO guidelines further specified that given the conflicting results with oxaliplatin, NalIRI, if available, should be the preferred regimen [10]. SEOM guidelines do not indicate a preference for any of the two regimens and emphasizes that none of them have been tested in patients treated with the combination of nab-paclitaxel + GEM or FOLFIRINOX in the first line [11]. For patients who have received fluoropyrimidine-based first line treatment, NCCN guidelines recommend a gemcitabine-based regimen in the second line setting [12]. Table 9 summarizes the current approach in the treatment sequence for advanced PDAC.

Table 9. Current approaches in treatment sequencing for mPDAC.

\begin{tabular}{|c|c|c|}
\hline First Line & Second Line & Third Line \\
\hline $\begin{array}{l}\text { GEM-based } \\
\text { (GEM, Combination of } \\
\text { nab-paclitaxel + GEM, } \\
\text { GEM-erlotinib) }\end{array}$ & $\begin{array}{l}\text { PS 0-1: Fluoropyrimidine based } \\
\text { regimen (+/- platinum and or } \\
\text { NalIRI); FOLFIRINOX } \\
\text { PS-2: Fluoropyrimidine alone; } \\
\text { BSC }\end{array}$ & $\begin{array}{l}\text { PS 0-1: } \\
\text { NalIRI or platinum } \\
\text { based regimen (if no } \\
\text { prior exposure) }\end{array}$ \\
\hline FOLFIRINOX & $\begin{array}{l}\text { PS 0-1: Combination of } \\
\text { nab-paclitaxel } \\
\text { PS-2: GEM monotherapy, BSC }\end{array}$ & ?? \\
\hline
\end{tabular}

It can be concluded that the treatment of patients with mPDAC has improved over the last few years with more effective chemotherapy regimens in first and second line settings. Both the combination of nab-paclitaxel + GEM and FOLFIRINOX showed improvement in survival in randomized phase III studies and are currently considered standard of care in the first line setting. Differences in study design, patients' characteristics, toxicity profile, resource consumption and supportive care required, affect the clinical application of these regiments in routine clinical practice. While no comparative study exists, the combination of nab-paclitaxel + GEM appears better tolerated, easier to administer and applicable to a broader patient population. The proportion of patients that are candidates to receive second and third line regimens is increasing due to better results with achieved with front line regimens. Management of MPDAC should be considered as a continuum care rather than a compartmentalized approach. In this sense, sequential treatment algorithms that interconnect first and subsequent lines are being developed.

\section{Acknowledgements}

The authors thank Sofía Perea, Pharm D, PhD, for her support in writing the manuscript.

\section{Funding}

The support for medical writing was provided by Fundación ECO.

\section{Competing Interests}

The authors have declared that no competing interest exists.

\section{References}

1. Skelton WP 4th, Parekh H, Starr JS, et al. Clinical Factors as a Component of the Personalized Treatment Approach to Advanced Pancreatic Cancer: a Systematic Literature Review. J Gastrointest Cancer. 2017 Nov 7. doi: 10.1007/s12029-017-0021-z.

2. Hidalgo M, Álvarez R, Gallego J, et al. Consensus guidelines for diagnosis, treatment and follow-up of patients with pancreatic cancer in Spain. Clin Transl Oncol. 2017;19:667-681

3. Martín AJ, Adeva J, Martínez-Galán J, et al. Pancreatic ductal adenocarcinoma: metastatic disease. Clin Transl Oncol. 2017;19:1423-1429.

4. Hidalgo M. Pancreatic cancer. N Engl J Med. 2010; 362: 1605-17.

5. NCCN. Clinical practice guidelines in oncology: pancreatic adenocarcinoma. Available

http://www.nccn.org/professionals/physician_gls/pdf/pancreatic.pdf. Accessed February 20, 2017.

6. Von Hoff DD, Ervin T, Arena FP, et al. Increased survival in pancreatic cancer with nab-paclitaxel plus gemcitabine. N Engl J Med. 2013; 369: 1691-1703.

7. Conroy $\mathrm{T}$, Desseigne $\mathrm{F}$, Ychou $\mathrm{M}$, et al. FOLFIRINOX versus gemcitabine for metastatic pancreatic cancer. N Engl J Med. 2011; 364: 1817-25.

8. Wang-Gillam A, Li C-P, Bodoky G, et al. Nanoliposomal irinotecan with fluorouracil and folinic acid in metastatic pancreatic cancer after previous gemcitabine-based therapy (NAPOLI-1): a global, randomised, open-label, phase 3 trial. Lancet. 2016;387:545-55.

9. Oettle H, Riess H, Stieler JM, Heil G, et al. Second-line oxaliplatin, folinic acid, and fluorouracil versus folinic acid and fluorouracil alone for gemcitabine-refractory pancreatic cancer: outcomes from the CONKO-003 trial. J Clin Oncol. 2014; 10;32:2423-29.

10. Ducreux M, Cuhna AS, Caramella C, et al. Cancer of the pancreas: ESMO clinical practice guidelines for diagnosis, treatment and follow-up. Ann Oncol. 2015; 26:v56-68.

11. Vera R, Ferrández A, Ferrer CJ, et al. Procedures and recommended times in the care process of the patient with pancreatic cancer: PAN-TIME consensus between scientific societies. Clin Transl Oncol. 2017;Jan 19 doi: 10.1007/s12094-016-1609-7.

12. Tempero MA, Malafa MP, Al-Hawary M, et al. Pancreatic Adenocarcinoma, Version 2.2017, NCCN Clinical Practice Guidelines in Oncology. J Natl Compr Canc Netw. 2017;15:1028-61

13. Sohal DP, Mangu PB, Khorana AA, et al. Metastatic Pancreatic Cancer: American Society of Clinical Oncology Clinical Practice Guideline. J Clin Oncol. 2016; 34:2784-96.

14. Burris HA 3rd, Moore MJ, Andersen J, et al. Improvements in survival and clinical benefit with gemcitabine as first-line therapy for patients with advanced pancreas cancer: a randomized trial. J Clin Oncol 1997;15:2403-13.

15. Moore MJ, Goldstein D, Hamm J, et al. Erlotinib plus gemcitabine compared with gemcitabine alone in patients with advanced pancreatic cancer: a phase III trial of the National Cancer Institute of Canada Clinical Trials Group. J Clin Oncol. 2007;25:1960-66

16. Ciliberto D, Botta C, Correale $\mathrm{P}$, et al. Role of gemcitabine-based combination therapy in the management of advanced pancreatic cancer: a meta-analysis of randomised trials. Eur J Cancer 2013;49:593-603. 
17. Waddell N, Pajic M, Patch AM, et al. Whole genomes redefine the mutational landscape of pancreatic cancer. Nature. 2015; 518:495-501.

18. Hidalgo M, Álvarez R, Gallego J, et al. Consensus guidelines for diagnosis, treatment and follow-up of patients with pancreatic cancer in Spain. Clin Transl Oncol. 2017;19:667-81.

19. Goldstein D, Chiorean EG, Tabernero J, et al. Outcome of second-line treatment following nab-paclitaxel + gemcitabine alone for metastatic pancreatic cancer. J Clin Oncol; 2016 34:333.

20. Tabernero J, Chiorean EG, Infante JR, et al. Prognostic factors of survival in a randomized phase III trial (MPACT) of weekly nab-paclitaxel plus gemcitabine versus gemcitabine alone in patients with metastatic pancreatic cancer. Oncologist 2015;20:143-50.

21. Tabernero J, Kunzmann V, Scheithauer W, et al. Nab-Paclitaxel plus gemcitabine for metastatic pancreatic cancer: a subgroup analysis of the Western European cohort of the MPACT trial. OncoT Ther. 2017:10 591-596.

22. Abrams TA, Meyer G, Moloney J, et al. Patterns of chemotherapy (CT) use in a population-based US-wide cohort of patients (pts) with metastatic pancreatic cancer (MPC). J Clin Oncol. 2014;32:abstr 4131.

23. Gourgou-Bourgade S, Bascoul-Mollevi C, Desseigne F, et al. Impact of FOLFIRINOX compared with gemcitabine on quality of life in patients with metastatic pancreatic cancer: results from the PRODIGE 4/ACCORD 11 randomized trial. J Clin Oncol. 2013; 31:23-39.

24. Chioeran G, Whiting S, Margunato-Debay S, Botteman M. Impact of nab-paclitaxel (nab-P) plus gemcitabine (G) vs Gemcitabine Alone on Karnofsky Performance Status (KPS) in Metastatic Pancreatic Cancer pts with Good or Poor Performance Status at Baseline: a Post-hoc Analysis of the MPACT Trial. ESMO 2015; abstr 2346.

25. Reni M, Wan Y, Solem C, Whiting S, Ji X, Botteman M. Quality-adjusted survival with combination nab-paclitaxel + gemcitabine vs gemcitabine alone in metastatic pancreatic cancer: a Q-TWiST analysis. J Med Econ. 2014; 17:338-46

26. Peixoto RD, Ho M, Renouf DJ, et al. Eligibility of Metastatic Pancreatic Cancer Patients for First-Line Palliative Intent nab-paclitaxel Plus Gemcitabine Versus FOLFIRINOX. Am J Clin Oncol. 2017;40:507-11.

27. Cartwright TH, Ginsburg A, Wilfong LS, Harrell R, Hoverman JR. Use of first line chemotherapy for advanced pancreatic cancer: FOLFIRINOX vs. gembitabine-based therapy. J Clin Oncol. 2014;32:abstr 4132

28. Braiteh F, Patel MB, Parisi M, Ni Q, Park S, Faria C. Comparative effectiveness and resource utilization of nab-paclitaxel plus gemcitabine (nab-P+G) versus FOLFIRINOX (FFX) in first-line treatment of advanced pancreatic adenocarcinoma (PDAC) in a U.S. community oncology setting. J Clin Oncol. 2016;34:abstr 433

29. Cubillo A, Dea A, Muñoz A, et al. YOSEMITE: A 3 Arm Double-Blind Randomized Phase 2 Study of Gemcitabine, Paclitaxel Protein-Bound Particles for Injectable Suspension (Abraxane ${ }^{\circledR}$ ) and Placebo (GAP) versus Gemcitabine, Abraxane ${ }^{\circledR}$ and either 1 or 2 Truncated Courses of Demcizumab. Ann Oncol. 2017;28:v209-v68.

30. Giordiano G, Vaccaro V, Lucchini E, et al. Analysis of prognostic factors in advanced pancreatic cancer (APDAC) patients (pts) undergoing to first line nab-paclitaxel (Nab-P) and gemcitabine $(G)$ treatment. J Clin Oncol. 2015;33:abstr 412 .

31. Giordiano G, Vaccaro V, Lucchini E, et al. Nab-paclitaxel (Nab-P) and gemcitabine (G) as first-line chemotherapy (CT) in advanced pancreatic cancer (APDAC) elderly patients (pts): A "real-life" study. J Clin Oncol. 2015;Suppl 3:abstr 424.

32. Giordiano G, Febbraro A, Vaccaro V, et al. Nab Paclitaxel (Nab-P) and Gemcitabine (G) as first line chemotherapy (CT) in advanced pancreatic cancer (APDAC) patients (pts): An Italian "real life" study. ECC. 2015:abstr 2334.

33. Baldini $\mathrm{C}$, Escande $\mathrm{A}$, Bouché $\mathrm{O}$, et al. Folfirinage: Tolerance and efficacy of folfirinox in elderly patients with advanced pancreatic adenocarcinoma. ECC. 2015;abst 1326.

34. Alessandretti MB, Moreira RB, Brandao EP, et al. Safety and efficacy of modified dose-attenuated FOLFIRINOX chemotherapy in patients over 65 years with advanced pancreatic adenocarcinoma. ASCO GI. 2015:abstr 468.

35. Maroun J, Ko YJ, Ghafoor A. A registry of real-world clinical practice on the use of FOLFIRINOX (FFX) in advanced pancreatic cancer (aPC) patients in Canada. ESMO. 2015; abstr 302P.

36. Gunturu KS, Yao X, Cong X, et al. FOLFIRINOX for locally advanced and metastatic pancreatic cancer: single institution retrospective review of efficacy and toxicity. Med Oncol. 2013; 30:361.

37. James ES, Cong X, Yao X, et al. Final analysis of a phase II study of Yale-modified FOLFIRINOX (mFOLFIRINOX) in metastatic pancreatic cancer (MPC). J Clin Oncol. 2015; 33:abstr 395.

38. Stein SM, James ES, Deng Y, et al. Final analysis of a phase II study of modified FOLFIRINOX in locally advanced and metastatic pancreatic cancer. Br J Cancer. 2016: 114:737-43.

39. Vivaldi C, Caparello C, Musettini G, et al. First-line treatment with FOLFOXIRI for advanced pancreatic cancer in clinical practice: Patients' outcome and analysis of prognostic factors. Int J Cancer. 2016; 139:938-45.

40. Vaccaro V, Sperduti I, Milella M. FOLFIRINOX versus Gemcitabine for Metastatic Pancreatic Cancer. N Engl J Med. 2011;365:768-9.

41. Watanabe K, Hashimoto Y, Umemoto K, et al. Clinical outcome of modified FOLFIRINOX versus gemcitabine plus nab-paclitaxel as first line chemotherapy in metastatic pancreatic cancer. J Clin Oncol. 2017;35:abstr 438.
42. Krishna K, Blazer MA, Wei L, et al. Modified gemcitabine and nab-paclitaxel in patients with metastatic pancreatic cancer (MPC): A single-institution experience. J Clin Oncol. 2015;33:abstr 366.

43. Scheithauer W, Ramanathan RK, Moore M, et al. Dose modification and efficacy of nab-paclitaxel plus gemcitabine vs. gemcitabine for patients with metastatic pancreatic cancer: phase III MPACT trial. J Gastrointest Oncol. 2016;7:469-73.

44. Fernandez-Montes A, Gonzalez Villarroel P, Valladares Ayerbes M, et al. Retrospective analysis of prognostic and predictive markers in patients with locally advanced unresectable and metastatic pancreatic adenocarcinoma treated with gemcitabine/nab-paclitaxel: Influence of the presence of stent. J Clin Oncol. 2015; 33:abstr 483

45. Giordiano G, Milella M, Lo Re G, et al. Nab paclitaxel (Nab-P) and gemcitabine $(G)$ first line chemotherapy $(C T)$ in metastatic pancreatic cancer $(\mathrm{mPC})$ patients (pts) relapsed after adjuvant treatment (ADJ T): A "real life" study. J Clin Oncol. 2017;35:Abstr 396.

46. Bouillard JB, Bouillaud S. De l'Obliteration des veines et de son influence sur la formation des hydropisies partielles: consideration sur la hydropisies passive et general. Arch Gén Méd. 1823;1:188-204.

47. Stein PD. Incidence of venous thromboembolism in patients hospitalized with cancer. Am J Med. 2006;119:60-8.

48. Epstein AS, Soff GA, Capanu M, et al. Analysis of incidence and clinical outcomes in patients with thromboembolic events and invasive exocrine pancreatic cancer. Cancer. 2012;118:3053-61.

49. Gary T, Belaj K, Steidl K, et al. Asymptomatic deep vein thrombosis and superficial vein thrombosis in ambulatory cancer patients: impact on short-term survival. Br J Cancer. 2012; 107:1244-8.

50. Piazza G, Goldhaber SZ. Management of submassive pulmonary embolism. Circulation. 2013; 122:1124-9.

51. Farge D, Bounameaux H, Brenner B, et al. International clinical practice guidelines including guidance for direct oral anticoagulants in the treatment and prophylaxis of venous thromboembolism in patients with cancer. Lancet Oncol 2016;17:e452-e66.

52. Shah MA, Ilson D, Kelsen DP. Thromboembolic events in gastric cancer: high incidence in patients receiving irinotecan- and bevacizumab-based therapy. J Clin Oncol. 2005; 23:2574-6.

53. Shah MA, Capanu M, Soff G, Asmis T, Kelsen DP. Risk factors for developing a new venous thromboembolism in ambulatory patients with non-hematologic malignancies and impact on survival for gastroesophageal malignancies. J Thromb Haemost. 2010;8:1702-9.

54. Ashrani AA, Guilerud E, Petterson TM, Marks RS, Bailey KR, Helt JA. Risk factors for incident venous thromboembolism in active cancer patients: A population based case-control study. Thromb Res. 2016;139:29-37.

55. Riess $H$, Habbel $P$, Jühling A, Sinn M, Pelzeret U. Primary prevention and treatment of venous thromboembolic events in patients with gastrointestinal cancers - Review. World J Gastroint Oncol. 2016;8:258-70.

56. Rothenberg ML, Meropol NJ, Poplin EA, Van Cutsem E, Wadler S. Mortality Associated With Irinotecan Plus Bolus Fluorouracil/Leucovorin: Summary Findings of an Independent Panel. J Clin Oncol 2001;9:3801-7.

57. Gaddh M, Antun A, Yamada K. Venous access catheter-related thrombosis in patients with cancer. Leukemia \& Lymphoma. 2014;55:501-508.

58. Castaman G. Risk of thrombosis in cancer and the role of supportive care (transfusion, chatheters, and growth factors). Thromb Res. 2016; 140:S89-92.

59. Verso M, Agnelli G, Barni S, Gasparini G, La Bianca R. A modified Khorana risk assessment score for venous thromboembolism in cancer patients receiving chemotherapy: the PROTECHT score. Intern Emerg Med. 2012; 7:291-2.

60. Saif MW, Shah MM, Shah AR. Fluoropyrimidine-associated cardiotoxicity: revisited. Expert Opin Drug Saf Hashmi S, Bell D, Diasio RB (2009) Prognostication of pancreatic adenocarcinoma by expression of thymidine phosphorylase/ dihydropyrimidine dehydrogenase ratio and its correlation with survival. Expert Opin Drug Saf. 2009; 8191-202.

61. Romá Sánchez E, García Pellicer J, Pelufo Pellicer A, Poveda AJL. Agencias de evaluación de tecnologías sanitarias. Utilidad de sus informes de evaluación en un servicio de farmacia. Farm Hosp 2005;29:290-6.

62. Tsavaris N, Kosmas C, Vadiaka M, et al. Cardiotoxicity following different doses and schedules of 5-fluorouracil administration for malignancy - a survey of 427 patients. Med Sci Monit 2002;8:PI51-7.

63. Abraxane Investigator Brochure. http://www.ema.europa.eu/docs/es_ES/ document_library/EPAR___Product_Information/human/000778/WC50002 0435.pdf, accessed May 31, 2017

64. 5-Fluorouracyl Investigator Brochure. https://www.aemps.gob.es/cima/ pdfs/es/ft/71868/71868_ft.pdf, accessed May 31, 2017

65. Chu E, de Vita T. Physicians' Cancer Chemotherapy Drug Manual. Jones and Bartlett Eds, 2017

66. Dominguez-Muñoz JE. Pancreatic Enzyme therapy for pancreatic exocrine insufficiency. Current Gastroenterology Reports. 2007;7:401-3.

67. Ihse, I, Lilja, P, Lundquist, I. Trypsin as a regulator of pancreatic secretion in the rat. Scand J Gastroenterol. 1979;14:873-80.

68. Partelli S, Frulloni L, Minniti C, et al. Faecal elastase-1 is an independent predictor of survival in advanced pancreatic cancer. Dig Liver Dis. 2012; 44:945-51.

69. Ellison NM, Chevlen E, Still CD, Dubagunta S. Supportive care for patients with pancreatic adenocarcinoma: symptom control and nutrition. Hematol Oncol Clin North Am. 2002; 16:105-21. 
70. Nagrial AM, Chin VT, Sjoquist KM, et al. Second-line treatment in inoperable pancreatic adenocarcinoma: A systematic review and synthesis of all clinical trials. CROH. 2015;96:483-97.

71. Chung V, McDonough S, Philip PA, et al. SWOGS 1115: Randomized Phase II clinical trial of Selumetinib (AZ6244, ARRY 142886) hydrogen sulfate (NSC-748727) and MK-2206(NSC-749607) vs. mFOLFOX in patients with metastatic pancreatic cancer after prior chemotherapy. SWOGS. 2015;abst 1115.

72. Gill J, Ko Y-J, Cripps MC, Beaudoin A, et al. PANCREOX: A randomized phase 3 study of 5FU/LV with or without oxaliplatin for second-line advanced pancreatic cancer (APC) in patients (pts) who have received gemcitabine (GEM)-based chemotherapy (CT). J Clin Oncol 2014;32:Abstr 4022.

73. Gill S, Ko YJ, Cripps C, et al. PANCREOX: A randomized phase iii study of fluorouracil/leucovorin with or without oxaliplatin for second-line advanced pancreatic cancer in patients who have received gemcitabine-based chemotherapy. J Clin Oncol. 2016; 34:3914-20.

74. Gilabert M, Rho YS, Kavan T, et al. Optimal therapeutic sequences in the treatment of metastatic pancreatic cancer- a retrospective analysis of two Canadian and French tertiary cancer centers. ECC. 2015; abstr 2359.

75. Giordano G, Febbraro A, Milella M. Impact of second line treatment (2l t) in advanced pancreatic cancer (APDAC) patients (pts) receiving first-line nab-paclitaxel (nab-p) + gemcitabine $(\mathrm{g})$ : an italian multicentre "real life" experience. ASCO. 2016; abstr 4124

76. Braiteh FS, Patel M, Parisi M, Ni Q, Park SY, Faria C. Comparative Effectiveness and Resource Utilization of nab-paclitaxel Plus Gemcitabine (nab-P + G) Versus FOLFIRINOX (FFX) in First-Line Treatment of Advanced Pancreatic Adenocarcinoma in a US Community Oncology Setting. ASCO GI. 2016; abstr 433.

77. Smith S, Jayavalsan SP, Durkal, et al. Can the sequence of therapeutic regimen influence outcome in patients with metastatic pancreatic carcinoma (MPAC)? ASCO GI. 2016; abstr 428

78. Chiorean EG, Von Hoff DD, Tabernero J, et al. Second-line therapy after nab-paclitaxel plus gemcitabine or after gemcitabine for patients with metastatic pancreatic cancer. Br J Cancer 2016;115:188-194.

79. Portal A, Pernot S, Tougeron D, et al. Nab-paclitaxel plus gemcitabine for metastatic pancreatic adenocarcinoma after Folfirinox failure: an AGEO prospective multicentre cohort. Br J Cancer. 2015;113:989-95. 